\title{
Del conocimiento a la práctica: integración de equipos de trabajo por supervisora de enfermería ${ }^{1}$
}

\author{
Danelia Gómez Torres $^{2}$, Karla Sofìa Gómez Alcántara ${ }^{3}$, Margarita Calderon Miranda ${ }^{4}$, Elizabeth Bernardino ${ }^{5}$
}

Institución: Universidad Autonoma del Estado de México, México.

\section{RESUMEN}

Esta investigación tuvo por objetivo determinar las estrategias de la enfermera supervisora en la integración de equipos de trabajo de enfermería en una institución hospitalaria. Se trata de una investigación cualitativa realizada en un hospital de segundo nivel de atención, entre agosto de 2017 y julio de 2018. Se aplicó una entrevista individual a 10 enfermeras supervisoras de dicha institución. El análisis se efectuó mediante la técnica cromática y el análisis de contenido; la discusión se elaboró con base en el contraste de los datos obtenidos con la teoría de los Roles de Equipo de Belbin. Se presentan en categorías y subcategorías relacionadas con las características del talento humano de enfermería: nivel de conocimientos, habilidades técnicas, personalidad, habilidades interpersonales y equipos de alto desempeño. Se identificaron las estrategias que las supervisoras de enfermería utilizan al integrar equipos de trabajo.

Palabras clave: Enfermería; Personal-de-enfermería; Supervisión-de-enfermería.

DOI 10.15517/revenf.v0i38.38385

${ }^{1}$ Fecha de recepción: 18 de julio del 2019

2 Enfermera. Doctora en Enfermeria. Profesorc

electrónico: gomezdanelia@usa.net

${ }^{3}$ Enfermera. Universidad Autónoma del Estado de México. Profesora en la facultad de Enfermería y Obstetricia de la UAEMÉX. México. Correo electrónico: karsof37@hotmail.com

${ }^{4}$ Enfermera. Doctora en Alta Dirección. Maestra en Administración. Profesora de la Facultad de Enfermería y Obstetricia de la UAEMÉX. México. Correo electrónico: calderonmcm@hotmail.com

${ }^{5}$ Enfermera. Doctora en Enfermería. Profesora adjunta del Departamento de Enfermería de la Universidad Federal de Paraná. Curitiba-

Paraná-Brasil. Correo electrónico: elizaber@ufpr.br 


\title{
From knowledge to practice: integration of work teams by nursing supervisor ${ }^{1}$
}

\author{
Danelia Gómez Torres ${ }^{2}$, Karla Sofia Gómez Alcántara ${ }^{3}$, Margarita Calderon Miranda ${ }^{4}$, Elizabeth Bernardino ${ }^{5}$
}

Institution: Autonomous University of the State of Mexico. Mexico.

\begin{abstract}
This research aimed to determine the strategies of the supervising nurse in the integration of nursing work teams in a hospital institution. This is a qualitative investigation carried out in a second level hospital, between August 2017 and July 2018. An individual interview was applied to 10 supervising nurses of that institution. The analysis was carried out using the chromatic technique and the content analysis; the discussion was developed based on the contrast of the data obtained with the Belbin Team Roles theory. They are presented in categories and subcategories related to the characteristics of human nursing talent: level of knowledge, technical skills, personality, interpersonal skills and high performance teams. Strategies that nursing supervisors use when integrating work teams were identified.
\end{abstract}

Keywords: Nursing; Nursing-staff; Nursing-supervisory.

DOI 10.15517/revenf.v0i38.38385

${ }^{1}$ Date of receipt: July 18, $2019 \quad$ Date of acceptance: October 31,2019
${ }^{2}$ Nurse. Doctor of Nursing. Professor at the Autonomous University of the State of Mexico (in Toluca). Mexico. gomezdanelia@usa.net

${ }^{3}$ Nurse.Autonomous Mexico State University. Professor in the Faculty of Nursing and Obstetrics of the UAEMÉX. Mexico. E-mail: karsof37@,hotmail.com

${ }^{4}$ Nurse. Doctor in Senior Management. Master in administration. Professor of the Faculty of Nursing and Obstetrics of the UAEMÉX.

Mexico. E-mail: calderonmcm@,hotmail.com

${ }^{5}$ Nurse. .Doctor of Nursing. Associate professor of the Department of Nursing of the Federal University of Paraná. Curitiba-Paraná.

Brazil. E-mail: elizaber@ufpr.br 


\section{Do conhecimento á pratica: integração de equipes de trabalho por supervisora de enfermagem ${ }^{1}$}

Danelia Gómez Torres ${ }^{2}$, Karla Sofia Gómez Alcántara ${ }^{3}$, Margarita Calderon Miranda ${ }^{4}$, Elizabeth Bernardino ${ }^{5}$

Instituição: Universidade Autônoma do Estado do México

\section{RESUMO}

Esta pesquisa teve como objetivo determinar as estratégias do enfermeiro supervisor na integração de equipes de trabalho de enfermagem em uma instituição hospitalar. Trata-se de uma investigação qualitativa realizada em um hospital de segundo nível, entre agosto de 2017 e julho de 2018. Foi aplicada uma entrevista individual a 10 enfermeiros supervisores dessa instituição. A análise foi realizada utilizando a técnica cromática e a análise de conteúdo; a discussão foi desenvolvida com base no contraste dos dados obtidos com a teoria dos papéis da equipe de Belbin. São apresentados em categorias e subcategorias relacionadas às características do talento humano em enfermagem: nível de conhecimento, habilidades técnicas, personalidade, habilidades interpessoais e equipes de alto desempenho. Estratégias que os supervisores de enfermagem usam ao integrar equipes de trabalho foram identificadas.

Palavras chave: Enfermagem; Recursos-humanos-de-enfermagem; Supervisão-de-enfermagem.

DOI 10.15517/revenf.v0i38.38385

${ }^{1}$ Data de recepção: 18 de julho de 2019

${ }^{2}$ Enfermeira Doutora em Enfermagem. Professor da Universidade Autônoma do Estado do México (em Toluca). México. Correio eletrônico: gomezdanelia@usa.net

3 Enfermeira Universidade Autônoma do Estado do México. Professora da Faculdade de Enfermagem e Obstetrícia da UAEMÉX. México. Correio eletrônico: karsof37@hotmail.com

4 Enfermeira Doutor em Gestão Sênior. Mestre em Administração. Professora da Faculdade de Enfermagem e Obstetrícia da UAEMÉX. México. Correio eletrônico: calderonmcm@hotmail.com

${ }^{5}$ Enfermeira Doutora em Enfermagem. Professora Associada do Departamento de Enfermagem da Universidade Federal do Paraná.

Curitiba-Paraná-Brasil. Correio eletrônico: elizaber@ufpr.br 


\section{INTRODUCCIÓN}

La supervisión en enfermería ha sido esencial para desempeñar procesos de gestión dentro de las instituciones hospitalarias. Las enfermeras que ejercen esta actividad se enfrentan constantemente a desafíos, en especial, en la gestión del talento humano; al respecto, la integración de equipos de trabajo ha sido una opción viable para hacerlo, puesto que los gerentes y líderes de las organizaciones deben aprender a discernir cuándo y para qué formar equipos; con el fin de generar y otorgar condiciones que faciliten el éxito antes, durante y después de terminar su misión ${ }^{1}$.

En este sentido, un equipo de trabajo representa más que una división de funciones, ya que su finalidad es conjuntar un determinado número de personas con diferentes habilidades, y dirigirlos a todos hacia el logro de un objetivo común. De esta forma, las organizaciones constituidas por seres humanos comparten actividades y responsabilidades para lograr sus propósitos, a través de la disposición de recursos y capacidades ${ }^{2}$.

No obstante, existen condiciones que dificultan a las supervisoras esta función, tal como: la plantilla de personal incompleta, no vigente o mal calculada que, en relación con el número de pacientes que el hospital atiende, ocasiona no disponer con un cuerpo de enfermería adecuado para realizar el trabajo. Asimismo, algunos de los factores que influyen, además de la falta de personal especializado en las diversas áreas del conocimiento, son las características individuales de las enfermeras; es decir, los rasgos de su personalidad, actitudes y valores; así como, las relaciones interpersonales que existen entre ellas y las normas institucionales; por ello:

La responsabilidad de los gestores de servicios de enfermería es asegurar la calidad asistencial a los usuarios así como las condiciones de trabajo a los profesionales de su equipo que han sido inhibidas tanto por la deficiencia numérica de los funcionarios como por la composición deficitaria del equipo de enfermería ${ }^{3}$.

Entonces, al aumentar el requerimiento de los servicios de enfermería, se deben buscar nuevas alternativas que lleven tanto al logro de objetivos, como al aumento de competitividad. Para ello, resulta necesario el rendimiento de los recursos, en especial, el talento humano, el cual representa un área de oportunidad por sus características únicas. Por consiguiente, para que el personal ofrezca su máximo desempeño, es necesario asignarle actividades en las que pueda aplicar sus conocimientos, habilidades y actitudes.

En esta línea, los equipos de trabajo son indispensables en las instituciones de salud; específicamente, los de enfermería, ya que, al reunir las habilidades de sus integrantes, se logra conjuntar esfuerzos y, así, obtener un trabajo más efectivo que lleve a consolidar los objetivos deseados. Asimismo, las estrategias para formar equipos deben adaptarse a los requerimientos institucionales y estar unidas a la innovación en la gestión institucional, de modo que permita considerar puntos importantes como: pensar en las condiciones de trabajo; detectar competencias, habilidades y capacidades; establecer expectativas y objetivos de desarrollo ${ }^{4}$.

Al realizar la función supervisora, la enfermera debe poseer habilidades tanto del ámbito gerencial como del asistencial; además de una característica formación profesional que le permita desempeñar el trabajo ${ }^{5}$ y organizar el talento humano en equipos de trabajo altamente calificados. Dichas habilidades son parte esencial de su deber profesional. 
El objetivo principal de esta investigación es revelar las estrategias que la enfermera supervisora debe seguir para integrar equipos de trabajo de enfermería en la institución hospitalaria.

\section{LA TEORÍA DE LOS ROLES DE EQUIPO DE BELBIN}

Se origina a partir de la necesidad de crear equipos eficaces de gran importancia para el funcionamiento de las organizaciones. Cabe mencionar que un equipo no es un conjunto de personas adscritas a determinados puestos de trabajo, sino que es una congregación de individuos en donde cada uno de ellos desempeña un rol entendido por el resto de miembros. Asimismo, los miembros se negocian entre sí el reparto de roles y llevan a cabo, de manera eficaz, los roles que les son más naturales, es decir, apoyados en sus habilidades complementarias ${ }^{6}$. Por tanto, el trabajo en equipo es el resultado de un conjunto de interrelaciones, conocimiento e información, actitudes y comportamientos que contribuyen en la dinámica de la productividad.

Los roles de equipo y los roles de trabajo. Para cumplir efectivamente con las tareas de un equipo, se requiere de integrantes que cumplan diversos roles; para de lograr esto, es necesario identificarlos como roles de equipo o de trabajo: este concepto se define como un patrón de conducta ${ }^{7}$ característico de la forma en que un miembro del equipo interactúa con otro, donde su desempeño facilita el progreso del grupo como un todo. Es decir, se compone de las características intrínsecas del individuo: comportamiento, personalidad e interrelación con otros.

Por consiguiente, los roles de trabajo o de funcionalidad son aquellas capacidades con las que cuenta un individuo para cubrir puestos de trabajo. En tal sentido, debe existir coherencia al asignar a los miembros del equipo, pues se deben adecuar los requisitos del puesto a las capacidades de los individuos y, a su vez, es necesario considerar capacidades técnicas, conocimiento técnico funcional y capacidad intelectual.

Por esta razón, un equipo ganador será aquel en el cual se representen ambos roles; es decir, miembros con características idóneas los asuman $\mathrm{y}$, en caso de carecer de ese rol, suplirlos con el fin de que no surjan contratiempos en la resolución de problemas ${ }^{6}$. Ahora, si se considera el perfil del trabajador como una mezcla de ambos roles y con el objetivo de integrarlos a un equipo de trabajo, es importante reflexionar acerca de varios factores.

Al diseñar un equipo, lo primero a tener en cuenta es la relación que existe entre los roles de equipo y roles funcionales. Un rol funcional aprovecha sus conocimientos técnicos y profesionales según lo requiera y un rol de equipo se describe como la forma de interactuar con los demás para facilitar el progreso común. El segundo se basa en la eficacia del equipo para adaptarse a los puntos fuertes, puesto que se elige el mejor rol que puede desempeñar cada miembro y se evita aquellos que limitan sus probabilidades de éxito. Además, un equipo debe aprovechar al máximo sus recursos técnicos, solamente cuando cuenta con los roles de equipo necesarios para garantizar un trabajo en equipo eficaz ${ }^{6}$.

En línea con lo anterior, los roles de equipo son cruciales para lograr efectividad en un grupo, pues deben mantener equilibrio con los roles funcionales y ser coherentes con las tareas asignadas al equipo y con los objetivos $^{8}$. De esta forma, un equipo puede desplegar sus recursos técnicos de manera óptima solo cuando existe un rango mínimo de roles de equipo que aseguren un trabajo de colaboración suficiente, ya que los equipos están 
organizados jerárquicamente $\mathrm{y}$, por tanto, deben cooperar y coordinarse a través de integrar, sintetizar y compartir información para lograr los propósitos ${ }^{7}$.

Por tanto, la efectividad de los equipos de trabajo está directamente relacionada con los distintos roles; puesto que la composición equilibrada de los equipos es el aspecto central que predice la efectividad de un equipo 9 .

\section{MATERIALES Y MÉTODO}

Para comenzar, el estudio se elaboró con un enfoque cualitativo a nivel descriptivo, ya que permite caracterizar un fenómeno concreto e indicar sus rasgos peculiares. Se aplicó el método de investigación convergente-asistencial que posibilita al investigador trabajar con pequeños grupos, con el objetivo de desarrollar la investigación y el crecimiento social y personal de las participantes e, incluso, incrementar la implementación de proyectos de práctica asistencial participativa, donde se hace abstracción de la práctica con la intención de construir conocimientos. Además, este método posibilita que los profesionales abandonen la postura directiva y tradicional y asuman una postura participativa en la práctica administrativa. El objeto del estudio fueron las estrategias que las enfermeras supervisoras utilizan para integrar equipos de trabajo en la institución hospitalaria.

De igual modo, la investigación se apoyó en la teoría de los roles de equipo, cuya posición metodológica es describir la manera de formar equipos ideales dentro de las organizaciones, al considerar sus necesidades y sus oportunidades. Para Belbin, esto se logra mediante la combinación adecuada de los roles funcionales y de equipo ${ }^{8}$, es decir, se considera el perfil de los individuos tanto en aspectos profesionales como personales. A partir de la teoría de Belbin, se obtuvieron los factores intervinientes/variables que guiaron la investigación: nivel académico, habilidades técnicas, nivel de conocimiento, personalidad y habilidades interpersonales, las cuales corresponden a roles funcionales y a roles de equipo.

Asimismo, la investigación tuvo como escenario un hospital de segundo nivel de atención, localizado en una entidad federativa mexicana tipo urbana. Se determinaron como actores sociales a 10 subjefes de enfermería, quienes conformaron la muestra considerada para esta investigación y cumplían los criterios de inclusión: enfermeras supervisoras con más de dos años de experiencia en la gestión del talento humano y con posgrado: maestría, doctorado o ambas. Con entrevistas obtenidas, se logró obtener la saturación de datos necesaria para cumplir con los objetivos del estudio.

En esta misma línea, los criterios de exclusión fueron: negativa de la supervisora a participar en la investigación o gozar de vacaciones durante el periodo de la recolección de datos. Además, respecto a los criterios de eliminación, fueron excluidas dos entrevistas que no contuvieron información relevante para el estudio.

En relación con el instrumento, lo constituyó una guía de entrevista semiestructurada, elaborada a partir de los factores que intervinieron en el estudio y se identificaron con base en los antecedentes teóricos de Belbin. Para ello, se generaron 10 preguntas conductoras de las que devinieron las once preguntas abiertas que conformaron el formato de entrevista final; además, se agregó un apartado que contenía una ficha de identificación con el número de entrevista y la fecha de realización. 
Cabe mencionar que previamente se aplicaron dos pruebas piloto para verificar que el instrumento era el adecuado para continuar con la investigación; las supervisoras, con quienes se llevó a cabo la prueba piloto, no eran trabajadoras de la misma institución, pero sí cumplían con los criterios de inclusión.

Una vez elaborado el instrumento, se recolectaron los datos mediante la técnica de entrevista, en el horario laboral de las supervisoras de enfermería; se acudió tanto a los centros de trabajo, como a las oficinas. Asimismo, el contacto para solicitar la entrevista se realizó personalmente y las enfermeras fueron grabadas con un equipo portátil, previa autorización de las participantes mediante el consentimiento informado. Posteriormente, las entrevistas se transcribieron para su análisis.

El análisis de contenido fue el método para estudiar los datos obtenidos, el cual contó con las siguientes fases. En primer lugar, el análisis previo, se dio una lectura reflexiva, en más de dos ocasiones, de las entrevistas transcritas, con el fin de seleccionar minuciosamente los fragmentos relevantes. En segundo lugar, se continuó con la fase de exploración en la que se examinó la totalidad del material a través del análisis de ocurrencia y concurrencia realizado sobre los temas; según el cual, el análisis de contenido generó una línea organizativa sobre la integración de los equipos de trabajo de enfermería, dividida en subcategorías. Esto facilitó la selección de unidades de mayor significado para el análisis sistemático de los datos.

En tercer lugar, se prosiguió con la fase de agrupación, en donde se identificaron las contribuciones al tema, lo que permitió generar las etiquetas para las categorías. Además, en apego a las preguntas principales de la investigación, se realizó de acuerdo con su similitud y complementariedad por medio de la técnica cromática. Por último, en la etapa de tratamiento final, se consideró, una vez más, el contenido de todas las entrevistas, para así, generar nuevas agrupaciones y corroborar que se hubiera dado respuesta a las preguntas iniciales de la investigación.

\section{Consideraciones éticas de la investigación}

De acuerdo con el artículo 14 del Reglamento de la Ley General de Salud en Materia de Investigación para la Salud, que establece: La investigación que se realice en seres humanos deberá desarrollarse conforme a las siguientes bases: [...] V. Deberá contar con el consentimiento informado y por escrito del sujeto de investigación $[\ldots],{ }^{10}$ se proporcionó un consentimiento informado que fue leído y firmado previamente por las participantes.

Además, como el Reglamento señala la obligación de proteger la privacidad de los participantes, se procedió a identificarlos mediante un número, con el fin de mantener su anonimato. Además, en la fecha 14 de septiembre de 2017, se envió, para su autorización, al comité de ética del Centro de Investigación y Ciencias Médicas de la Universidad Autónoma del Estado de México, con el propósito dar cumplimiento a la normatividad y a los lineamientos éticos.

\section{RESULTADOS}

Si bien, para integrar los equipos de alto desempeño se requieren personas con cierto nivel de efectividad, no serán las competencias de sus miembros lo que determine el nivel de desempeño; pues, este se definirá, 
principalmente, por la sinergia que se logre configurar entre los miembros, el grado de especialización que se defina en las tareas, la confianza que se logre construir entre los sujetos y las habilidades como grupo en torno a la motivación y a la comunicación efectiva. Por ello, las supervisoras establecen algunas pautas para integrar este modelo de equipos de trabajo y se exponen los hallazgos_-según emergieron— por categorías:

\begin{tabular}{|c|c|}
\hline CATEGORÍA & HALLAZGOS \\
\hline EQUIPOS DE ALTO DESEMPEÑO & $\begin{array}{l}\text { Los equipos de alto rendimiento, los puedes } \\
\text { organizar cuando tienen todos el mismo } \\
\text { objetivo, cuando dan más de lo que les } \\
\text { marca su propia categoría, el equipo de alto } \\
\text { rendimiento es cuando en lugar de dar } \\
100 \% \text { das } 110 \% \text { y todo para cumplir de } \\
\text { forma oportuna tus objetivos, para que se } \\
\text { cumplan de forma eficaz, eficiente al } \\
\text { máximo y tú le vas a exigir a esa persona } \\
\text { más de lo que es capaz de dar y te va a dar } \\
\text { excelentes resultados (E-2). } \\
\text { Para los gerentes o líderes de equipos uno } \\
\text { de los principales retos es la formación de } \\
\text { grupos de trabajo de alto desempeño; para } \\
\text { lograrlo, se debe crear un ambiente de } \\
\text { confianza, liderazgo, buena comunicación, } \\
\text { establecer un claro entendimiento del } \\
\text { objetivo a lograr y la participación de cada } \\
\text { miembro tratando de aprovechar al máximo } \\
\text { sus fortalezas, eso se refleja: } \\
\text { Para tener un equipo de alto rendimiento } \\
\text { debes hacer un diagnóstico de la gente con } \\
\text { la que vas a trabajar, porque si conoces sus } \\
\text { debilidades te vas a enfocar en } \\
\text { potencializarlas y transformarlas en } \\
\text { fortalezas, porque si minimizas la debilidad } \\
\text { y enalteces la fortaleza, entonces estás } \\
\text { haciendo que ese compañero rinda más y si } \\
\text { le comunicas cuáles son los objetivos y } \\
\text { haces ver que tiene la capacidad de dar más } \\
\text { de lo que él esperaba, tal vez mejore (E-2). }\end{array}$ \\
\hline
\end{tabular}

En este sentido, la personalidad de las enfermeras es fundamental para asegurar el funcionamiento de los equipos de trabajo, ya que no basta con movilizar sus recursos intelectuales, físicos o de interrelación, con la esperanza de incrementar su productividad. Esto debido a que lo que se requiere es comprometer a las personas en relación con 
el trabajo; por eso, las supervisoras de enfermería emplean estrategias encaminadas a lograrlo, tal como se refleja a continuación:

\begin{tabular}{|l|l|}
\hline CATEGORÍA & \multicolumn{1}{|c|}{ HALLAZGOS } \\
\hline PERSONALIDAD & $\begin{array}{l}\text { Yo creo que, en la actitud personal, porque } \\
\text { tenemos personal muy negativo y positivo } \\
(\ldots) \text { la actitud en muchas ocasiones -lo } \\
\text { podemos hacer- pero no queremos (E-2). } \\
\text { Buscamos quien tenga una personalidad de } \\
\text { servicio, se considera la actitud y aptitud } \\
\text { para que pueda desarrollar bien el trabajo } \\
\text { (..) una buena actitud de saber hacer y eso } \\
\text { te da la humildad para relacionarte con los } \\
\text { demás (E-2). } \\
\text { Tenemos como primer reto ganarnos al } \\
\text { personal, el segundo conocer cuál es el } \\
\text { carácter de cada una de ellas (E-7). } \\
\text { Quién tenga el carácter y la disposición de } \\
\text { realizar el trabajo en equipo (E-3). } \\
\text { No voy a dejar a personas de un mismo } \\
\text { carácter juntas porque entonces ya no } \\
\text { funcionarían en equipo (E-5). }\end{array}$ \\
\hline
\end{tabular}

Dichas opiniones, se refieren a las conductas que facilitan el logro de objetivos en situaciones de interacción al unir esfuerzos; no son conceptuados por un rasgo de la personalidad, sino comportamientos adquiridos. Por esta razón, cuanto mayor sea la unidad de los integrantes del equipo, más ligera será la división del trabajo; pues, cuando las personas lo comparten, las diferencias naturales que se constatan en los roles de equipo pueden resolver el problema de identidad, al tiempo que aumentan la sinergia productiva. Esta habilidad se ve reflejada en las siguientes aportaciones: 


\begin{tabular}{|l|l|}
\hline \multicolumn{1}{|c|}{ CATEGORÍA } & \multicolumn{1}{|c}{ HALLAZGOS } \\
\hline HABILIDADES INTERPERSONALES & $\begin{array}{l}\text { Deben tener una buena comunicación y } \\
\text { aprender a comunicarse, lo cual implica } \\
\text { saber escuchar para saber hacer (...) } \\
\text { conocer lo que quieren transmitir y qué } \\
\text { debo transmitir yo, para que el que se va a } \\
\text { interconectar conmigo tenga el mismo } \\
\text { objetivo... (E-2). } \\
\text { Una de las características es saber } \\
\text { interconectarse con los demás, saber en qué } \\
\text { va el compañero para que yo pueda también } \\
\text { tomar la parte que me corresponde, y así } \\
\text { trabajar conjuntamente en la función que } \\
\text { nos fue asignada (E-2). } \\
\text { Las habilidades interpersonales que se } \\
\text { consideran son empatía, comunicación, } \\
\text { negociación o manejo de conflictos, y } \\
\text { motivación invariablemente (E-1). }\end{array}$ \\
\hline
\end{tabular}

Además, las habilidades técnicas influyen en el rol que cada integrante del equipo desempeña, ya que logran hacerlo experto en un área, al ser parte de las fortalezas que puede aportar al equipo, debiendo tener una potenciación continua de las mismas. Por ello, las habilidades facilitan la adaptación a los equipos y mejoran los resultados, como se muestra enseguida:

\begin{tabular}{|l|l|}
\hline \multicolumn{1}{|c|}{ CATEGORÍA } & \multicolumn{1}{c|}{ HALLAZGOS } \\
\hline \multirow{3}{*}{ HABILIDADES TÉCNICAS } & $\begin{array}{l}\text { Las habilidades son muy importantes } \\
\text { porque esto hará que el equipo funcione (E- }\end{array}$ \\
& 5 ). \\
& Es muy importante que las enfermeras \\
tengan la habilidad de hacer las cosas, al \\
menos las técnicas básicas (E-8). \\
La técnica es indispensable porque puedes \\
tener todo el conocimiento, pero si no sabes \\
cómo hacerlo, entonces nada tienes (E-2). \\
Identificar las habilidades con las que \\
cuentan, pues esto les facilita adaptarse a \\
los servicios (E-8).
\end{tabular}

En esta línea, la enfermería se reconoce como una disciplina profesional, cuya esencia es la práctica sustentada en el conocimiento científico, considerado en los últimos años como un recurso indispensable para la producción de servicios, tal como se refleja en las siguientes declaraciones: 


\section{CATEGORÍA}

CONOCIMIENTOS

\section{HALLAZGOS}

Los conocimientos son muy importantes porque a través de ellos, ellas [las enfermeras] se apegan a la normatividad, aplican los procesos como debe ser (E-5). Los conocimientos son fundamentales porque tienes una razón del porqué se tiene que hacer así, debes tener un conocimiento claro del proceso, tienes que basarlo y sustentarlo... (E-2).

Es fundamental, es básico, eso tiene que obtenerse desde la carrera, todo eso ya es prácticamente de nosotros, es venir a aplicar sus conocimientos que ya adquirieron en las aulas (E-7).

Como se observa, en los equipos de trabajo la inteligencia forma parte esencial de aprovechar el talento humano y de utilizarlo eficazmente en campos fundamentales del ejercicio profesional de enfermería. En consecuencia, las estrategias de gestión deben ir encaminadas a potenciar las fortalezas, disminuir las debilidades y mantener la motivación mediante el liderazgo de la enfermera supervisora.

\section{DISCUSIÓN}

\section{Equipos de alto desempeño}

Al hablar de un equipo de alto desempeño no solo se considera la capacidad intelectual de las personas que lo conforman, sino también los diferentes conocimientos y competencias que, mediante la interacción, logran complementarse y generar satisfacción para todos. En este sentido, el logro de objetivos optimiza el trabajo, pues, sigue una relación esfuerzo-tiempo, lo cual da como resultado una fuerte motivación para mantener el ritmo de trabajo.

Ahora bien, uno de los retos en la gestión actual del talento humano es conformar equipos de trabajo de alto desempeño. Las instituciones de salud, debido a la importancia de los equipos, los requieren para aumentar la productividad y la calidad de los servicios, así como, para aumentar el desempeño del personal ${ }^{11}$. En consecuencia, las supervisoras de enfermería conocen la importancia de generar equipos de alto desempeño. Para Belbin ${ }^{12}$, esto se relaciona con la exigencia de formar equipos bien equilibrados, mediante la disponibilidad de candidatos adecuados con diversidad de talento y roles de equipo; quien los dirija debe considerar además su temperamento. Sin embargo, esto no asegura que la organización pueda cambiar por completo, en especial, respecto a sus reglas y estructuras.

Ante esto, las supervisoras de enfermería han entendido que para avanzar en las estrategias de gestión de talento humano en la institución hospitalaria, se requiere integrar al personal en equipos de trabajo que propicien un 
equilibrio entre las capacidades y el comportamiento de las enfermeras, donde cada una de sus integrantes asuma la responsabilidad de desempeñar su rol y participar activamente con los demás, imbuidas en un sentido de solidaridad y de apoyo que contribuya al logro de objetivos.

\section{Personalidad}

La personalidad se define como un conjunto de rasgos y cualidades que determinan la manera de ser de una persona y los valores que guían la conducta de los individuos. Estos rasgos y cualidades pueden llevar a comportamientos de liderazgo que, al ser percibidos por otros, originan el surgimiento de líderes en los equipos ${ }^{13}$.

Las características que implican la personalidad son un factor importante para la gestión del talento humano. Para Belbin $^{14}$, la personalidad es esencial en la integración de equipos, debido a que forma parte de los roles de equipo y es el principal tema de su teoría. En uno de sus hallazgos, refiere que, dentro de los equipos con integrantes de personalidades similares, no se obtienen efectos favorables respecto a la consecución de los objetivos del equipo.

En esta línea, las enfermeras supervisoras pueden mejorar la gestión del talento humano una vez identificadas las características personales del equipo de enfermería; principalmente, aquellas actitudes que se presentan ante diversas situaciones, pues de estas dependerá el rol de equipo que asuman. Es decir, el rol puede ser cambiante en relación con las necesidades existentes y la actitud permite adaptarse o no al equipo. Además, es preferible no incluir en un mismo equipo a personas con rasgos y cualidades similares, especialmente respecto al carácter, ya que se podrían generar conflictos que influirían de forma negativa en la productividad del equipo.

\section{Habilidades interpersonales}

El desempeño de cualquier rol profesional exige conocer, así como, dominar-junto a las capacidades conceptuales - un conjunto de habilidades sociales que permitan al profesional crear una relación eficaz y satisfactoria con los demás. Para una buena competencia de relaciones humanas, es necesaria una óptima comunicación interpersonal $^{15}$, tal como las participantes lo afirman.

En este sentido, la comunicación interpersonal se considera generadora de estrategias enfocadas a establecer equipos funcionales. Se le define como aquella en que se enfatiza la relación con el otro ${ }^{16}$, dicha relación no se puede pensar sin personalización, intimidad, empatía y confianza; además, no tiene que darse, necesariamente, a través de la proximidad física, sino en función de una proximidad afectiva que se recrea desde una posición ética. Lo anterior está relacionado de manera directa con el comportamiento que se define por lo que se hace y se dice ante una situación; es decir, corresponde, esencialmente, a cómo nos relacionamos con los otros y a la base de cómo se relacionan los demás ${ }^{14}$.

Por tanto, para que los equipos de trabajo de Enfermería sean funcionales, es fundamental que sus integrantes posean ciertas habilidades interpersonales que garanticen una adecuada interacción. Entonces, los equipos requieren de participantes capaces de comunicarse de forma clara y precisa-imprescindible para mantener una real colaboración con los demás-, de tal forma que la relación con los otros favorezca incrementar la productividad laboral y reducir conflictos. 


\section{Habilidades técnicas}

Son aquellas que se refieren a la capacidad que tienen las personas para aplicar métodos, procedimientos y técnicas de un área del conocimiento específica y que pueden ser especializadas ${ }^{17}$. En los equipos de trabajo, se usan las habilidades técnicas de sus integrantes para alcanzar los objetivos del equipo; de ahí, la importancia de identificarlas y desarrollarlas. Además, estas habilidades implican talento para realizar determinadas tareas; sin embargo, no significa que se tenga aptitud para realizar otro tipo de funciones, por tanto, el desarrollo de la habilidad es una parte de competencia para cumplir con una meta específica ${ }^{18}$. Las supervisoras implementan estrategias, para que las habilidades técnicas del personal de Enfermería sean empleadas de manera óptima en la conformación de los equipos de trabajo.

Lo anterior, hace patente que las habilidades técnicas de las enfermeras son indispensables para que se integren a un equipo específico, en el cual puedan aplicar sus habilidades con el fin de lograr objetivos. También, se requiere que el personal se complemente con los demás integrantes, ya que cada uno posee habilidades distintas que, finalmente, harán funcional al equipo y lo llevarán a conseguir sus metas, lo cual se reflejará en la calidad de atención a los pacientes.

\section{Conocimientos}

El conocimiento es un cúmulo de información interpretada en la realidad que la persona posee, puede ser adquirido de forma científica o empírica. Ahora bien, el conocimiento científico se fundamenta en el propio de enfermería derivado de los resultados de su investigación, de un cuerpo teórico propio y de otras disciplinas, aplicado y adaptado a la práctica, que conforman la esencia disciplinar. ${ }^{19}$ Debido a su importancia, la gerencia actual requiere de los conocimientos que la enfermera adquiere en la formación; por lo cual es necesario poseer conocimientos en el campo de la gestión.

En este sentido, se puede afirmar que las personas son valoradas por sus conocimientos, sus aportes intelectuales y su talento en la consecución de las metas de las organizaciones ${ }^{20}$;por eso, las supervisoras de enfermería aplican estrategias que permiten fundamentar las acciones en los conocimientos del personal.

En el ámbito de gestión, donde existe un sinnúmero de reglas complejas, limitaciones, variables y posibilidades, se presenta una situación en donde se valora altamente el conocimiento e inteligencia ${ }^{12}$. Sin embargo, el personal que desempeña los roles no encaja fácilmente en un sistema y requiere de una persona que le dirija; es decir, de un jefe con habilidad de descubrir el talento de estas personas y saber cómo y cuándo utilizarlo. Además, si el jefe es comprensivo y amable, pondrá de manifiesto lo mejor de aquellos con alto nivel de conocimientos ${ }^{21}$.

Finalmente, el conocimiento que debe poseer el personal de Enfermería es indispensable para desempeñarse dentro de un área específica; de igual modo, es esencial que dentro de un equipo de trabajo los integrantes realicen la práctica sustentada en el conocimiento científico, ya que el éxito de este tipo de equipos depende, en gran medida, de la capacidad de las supervisoras para identificar y saber utilizar el conocimiento de su personal. 


\section{CONCLUSIONES}

Se identificaron estrategias de supervisión relacionadas con factores que consideran las supervisoras, con el fin de conformar grupos de trabajo que incluyan las características del talento humano, guidas por aspectos relacionados con la formación profesional de las enfermeras: conocimientos y habilidades técnicas.

Además, se detectó que las supervisoras dan mayor importancia a las características de la personalidad y las habilidades interpersonales para integrar equipos, lo cual refleja un aporte científico a la gestión del talento humano en Enfermería; pues, la posiciona en un alto nivel, ya que se gestiona como en otros ámbitos. Asimismo, se destaca que en la práctica aplican los postulados que menciona la teoría de Belbin.

Del mismo modo, se obtuvo una categoría no prevista en los factores que intervinieron en el estudio: la categoría de equipos de trabajo de alto desempeño, lo cual refleja que las supervisoras de enfermería no solo aplican los conocimientos esenciales de gestión, sino que consideran oportunidades mediante la formación de dichos equipos; por lo cual, innovan en la gestoría del talento humano y, a su vez, tal nueva categoría es un hallazgo sobresaliente de la investigación.

Declaración de conflicto de intereses. No existe conflicto de intereses de las autoras.

\section{REFERENCIAS BIBLIOGRÁFICAS}

1. Malpica R, Rossell R, Hoffmann I. Equipos de trabajo de alto desempeño. Observatorio Laboral Revista Venezolana. 2014; 7(14): 69-83. Disponible en: https://www.redalyc.org/pdf/2190/219040849005.pdf

2. Sánchez S, Herrera M. Los recursos humanos bajo el enfoque de la teoría de los recursos y capacidades. Investigación y Reflexión. 2016; (24): 133-146. DOI: http://dx.doi.org/10.18359/rfce.2216

3. Soares I, Rodrigues ZM, Henriques S, De Souza Terra F. Gestión de recursos humanos y su interfaz en la sistematización de la asistencia de enfermería. Enfermería Global. 2016; 15 (2): 341-352. Disponible en: http://scielo.isciii.es/pdf/eg/v15n42/administracion3.pdf

4. Dias-Silveira C, Teixeira-de-Bessa T, Oroski-Paes A, Conceição-Stipp G, Andrade M. Gestión del equipo de enfermería: factores asociados a la satisfacción en el trabajo. Enfermería Global. 2017; 16 (3): 193-207. DOI: http://dx.doi.org/10.6018/eglobal.16.3.249471

5. Villegas-Gutiérrez NB, Soto-Arreola M, Meljem-Moctezuma J, Perez J. Perfil del supervisor de enfermería en la unidad de alta especialidad. Revista CONAMED. 2018; 23 (1): 28-32. Disponible en: https://dialnet.unirioja.es/servlet/articulo? codigo $=6372045$

6. Simón A. Estudio de los roles de equipo en el sector de la auditoría contable. España: Universidad Politécnica

de Valencia; 2015.

Disponible en: 
https://riunet.upv.es/bitstream/handle/10251/53309/SIM\%c3\%93N\%20-

\%20ESTUDIO \%20DE\%20LOS\%20ROLES\%20DE\%20EQUIPO\%20EN\%20UNA\%20EMPRESA \%20

DEL\%20SECTOR\%20DE\%20LA\%20AUDITOR\%c3\%8dA\%20CONTABLE..pdf?sequence=4\&isAllow ed $=y$

7. Rodríguez-Ponce E, Pedraja-Rejas L, Ganga-Contreras F. La relación entre los estilos de liderazgo y el desempeño de los equipos de dirección intermedia: un estudio exploratorio desde Chile. Contabilidad y Negocios. 2017; 12(23): 129-144. DOI: https://doi.org/10.18800/contabilidad.201701.009

8. Belbin M. Beyond the team. 2nd ed. New York: Routledge; 2012.

9. Sánchez OS, Herrera AM. Los recursos humanos bajo el enfoque de la teoría de los recursos y capacidades. Revista Facultad de Ciencias Económicas. 2016; 24(2): 133-146. DOI: http://dx.doi.org/10.18359/rfce.2216.

10. Secretaría de Salud. Reglamento de la Ley General de Salud en Materia de Investigación en Salud. Diario Oficial de la Federación. México; $1987 . \quad$ Disponible en: http://www.ijc.gob.mx/arc/8/11/d/RegLeySaludMateriaInvestigaci\%C3\%B3n.pdf

11. Rojas Ramírez C, Carlisbeth C. Conocimientos sobre competencias gerenciales en enfermeras supervisoras del Hospital Universitario Dr. Ángel Larralde. [Disertación]. Universidad de Carabobo; 2018. Disponible en: http://www.riuc.bc.uc.edu.ve/bitstream/123456789/8175/1/cramirez.pdf

12. Belbin M. Team roles at work. 2nd ed. New York: Elsevier; 2010.

13. Coluccio G, Rodríguez-Ponce E. Personalidad y el surgimiento de liderazgo en equipos: un estudio en estudiantes universitarios. Revista de ciencia y tecnología de América. 2018; 43(10): 723-728. Disponible: https://dialnet.unirioja.es/servlet/articulo?codigo $=6575865$

14. Belbin M. La guía de Belbin para triunfar en el trabajo. Inglaterra: Belbin Associates; 2009.

15. Flores E, García M, Calsina W, Yapuchura A. Las habilidades sociales y la comunicación interpersonal de los estudiantes de la Universidad Nacional del Altiplano. Revista de Investigación en Comunicación y Desarrollo. 2016; 7(2): 5-14. Disponible en: http://www.scielo.org.pe/pdf/comunica/v7n2/a01v7n2.pdf

16. Romeu V. Hacia una teoría personalista de la comunicación interpersonal. Revista especializada de periodismo y comunicación. 2015; 1 (48): 188-198. Disponible: file:///Users/ccubero/Downloads/2692Texto\%20del\%20art\%C3\%ADculo-10479-1-10-20151221.pdf

17. Instituto Tecnológico y de Estudios Superiores de Monterrey [Internet]. El desarrollo de habilidades, valores y actitudes propuestos en la misión; $2018 . \quad$ Disponible en: http://sitios.itesm.mx/va/dide2/documentos/havs.pdf 
18. López P, Díaz Z, Segredo A, Pomares Y. Evaluación de la gestión del talento humano en entorno hospitalario cubano. Revista Cubana de Salud Pública. 2017; 43(1): 3-15. Disponible en: http://scielo.sld.cu/pdf/rcsp/v43n1/spu02117.pdf

19. Durán de Villalobos M. Una mirada hacia afuera: proyección de enfermería para el 2050. Aquichan. 2018; 18(1): 5-8. DOI: 10.5294/aqui.2018.18.1.1

20. Majad Rondón M. Gestión del talento humano en organizaciones educativas. Revista de Investigación. 2016; 40(88): 148-165. Disponible en: http://ve.scielo.org/pdf/ri/v40n88/art08.pdf

21. Trespalacios M. Trabajo en equipo, especialidades formativas. Madrid: EditorialCEP:2017 